\title{
Classificadores Não-Supervisionados baseados em Conjuntos Fuzzy e Nuvens de Dados para Detecção de Spam
}

\author{
Eduardo Soares* Cristiano Garcia* Ricardo Pouças* \\ Stella Marys Lamounier ${ }^{* *}$ Daniel Leite* \\ * Departamento de Automática, Universidade Federal de Lavras, \\ UFLA, Brasil (edu.soares999@gmail.com, cristiano.garcia@ufla.br, \\ ricardo.poucas@gmail.com,daniel.leite@ufla.br). \\ ** Departamento de Computação, Centro Universitário UNA, Bom \\ Despacho,Brasil (stella.lamounier@gmail.com).
}

\begin{abstract}
:
Technological advancements have made individuals and organizations more dependent on e-mails to communicate and share information. The increasing use of e-mails has led to an increased production of unsolicited commercial messages, known as spam. Spam classification systems able to self-adapt over time, with no human intervention, are rare. Adaptation is interesting as spams vary over time due to the use of different message-masking techniques. Moreover, classification models that handle large volumes of data are essential. Evolving intelligent systems are able to adapt their parameters and structure according to the data stream. This study applies the evolving methods TEDA (Typicality and Eccentricity based Data Analytics) and FBeM (Fuzzy Set-Based Evolving Modeling) for online unsupervised classification of spams. TEDA and FBeM are compared in terms of accuracy, model compactness, and processing time. For dimensionality reduction, a non-parametric Spearman-correlation-based feature selection method is employed. A dataset containing 25,745 samples, being 7,830 spams and 17,915 legitimate e-mails, is considered. 711 features extracted from an e-mail server describe each sample.

Resumo: O avanço de tecnologias tem tornado indivíduos e organizações mais dependentes de e-mails para comunicação e compartilhamento de informação. O uso crescente de e-mail tem levado à produção de mensagens comerciais não-solicitadas, conhecidas como spam. Sistemas de classificação de spams capazes de se adaptar ao longo do tempo, sem intervenção humana, são raros. A adaptação é interessante já que spams variam no tempo devido ao uso de diferentes técnicas de mascaramento de mensagens. Além disso, modelos classificadores que lidam com grandes volumes de dados são essenciais. Sistemas inteligentes evolutivos são capazes de adaptar seus parâmetros e estrutura de acordo com o fluxo de dados. Este estudo aplica os métodos evolutivos TEDA (Typicality and Eccentricity based Data Analytics) e FBeM (Fuzzy SetBased Evolving Modeling) para classificação não-supervisionada de spam. TEDA e FBeM são comparados em termos de acurácia, compactação do modelo, e tempo de processamento. Para redução da dimensionalidade, um método não-paramétrico baseado em correlação de Spearman é empregado. Uma base de dados contendo 25745 amostras, sendo 7830 spams e 17915 e-mails legítimos, foi elaborada. 711 atributos extraídos de um servidor descrevem as amostras.
\end{abstract}

Keywords: Unsupervised Learning; Spam Detection; Evolving Intelligent Systems; Clustering; Data Stream.

Palavras-chave: Aprendizado Não-Supervisionado; Detecção de Spam; Sistemas Inteligentes Evolutivos; Clusterização; Fluxo de Dados.

\section{INTRODUÇÃO}

Com os avanços recentes das tecnologias de informação e de redes, organizações e indivíduos dependem cada vez mais de e-mails para se comunicarem e para compartilhar informações. No entanto, a crescente utilização de e-mails acarretou problemas causados por mensagens não solicitadas, conhecidas como spams. O envio de spam através de e-mails sobrecarrega servidores SMTP (Simple Mail Transfer Protocol), desperdiça banda, aborrece usuários, e gera prejuízos morais e até financeiros (Olivo et al., 2015). Devido à grande quantidade de técnicas de envio e mascaramento de mensagens por parte de spammers e às diferenças do conteúdo da mensagem que variam com a cultura, época, entre outros, o problema de detecção de spams está longe de ser solucionado.

De acordo com (Whitworth \& Whitworth, 2004), em uma escala global, a porcentagem de mensagens spam chega a $95 \%$ do total de mensagens trafegadas. Já um estudo divulgado pela Symantec revelou que no ano de 2010 o 
percentual de spams foi de $89,1 \%$ do número total de emails (Fossi et al., 2011). Em 2010, a quantidade registrada de mensagens spam foi de cerca de 62 bilhões (Fossi et al., 2011).

O problema de classificação de e-mails de texto se refere à discriminação entre spams e e-mails legítimos. Um sistema anti-spam verifica as palavras contidas no cabeçalho e no corpo do e-mail e compara se as mesmas ocorrem com certa frequência em mensagens spam típicas. Em geral, faz-se uso de uma base de dados contendo palavras recorrentes em spams (Olivo et al., 2015). Tal técnica é, por vezes, ineficaz visto que os spammers trocam caracteres corretos por outros, mantendo o sentido original. Isso confunde sistemas classificadores já que a combinação de caracteres é diferente.

De um modo geral, métodos de reconhecimento de padrões (Bishop, 1995) (Duda et al., 1995) têm sido investigados para classificação de spam. Estes métodos têm obtido taxas relativamente altas de classificações corretas em contexto estacionário. Ambientes não-estacionários requerem a adaptação de parâmetros de modelos classificadores a partir do uso de algoritmos incrementais de aprendizado de máquina. A idéia é que modelos classificadores acompanhem as mudanças dos padrões de spam ao longo do tempo (Soares et al., 2018) (Leite et al., 2012).

No presente trabalho são considerados modelos inteligentes evolutivos baseados em nuvem de dados - Typicality and Eccentricity Data Analytics (TEDA) (Soares et al., 2018) - e em grânulos e regras fuzzy - Fuzzy-Set-Based evolving Modeling (FBeM) (Leite et al., 2012) - para classificação de e-mails de texto, legítimos e spams. TEDA conta com um método de aprendizado que se baseia nos conceitos de nuvem de dados, excentricidade e tipicidade. A idéia é que nuvens TEDA não têm um formato geométrico específico, como clusters convencionais. FBeM usa objetos fuzzy granulares para sumarizar a informação extraída de um fluxo de dados. FBeM é baseado no conceito de cobertura (granulação fuzzy) do espaço dos dados. Suas regras são interpretáveis linguisticamente e, portanto, úteis para auxílio à tomada de decisão (Garcia et al., 2019).

Os métodos TEDA e FBeM são comparados em termos de acurácia de classificação, tempo de processamento e tamanho da base de regras gerada. Ademais, um método de redução da dimensionalidade dos dados baseado na correlação de Spearman, conforme (Soares et al., 2018), foi considerado para ordenação e seleção dos atributos mais discriminativos de classes. Portanto, as contribuições deste trabalho são as seguintes:

- aplicação de métodos de inteligência artificial, interpretáveis e adaptáveis, para a classificação de e-mails legítimos e spams de forma online;

- utilização de um seletor de atributos baseado na correlação de Spearman para a redução da dimensionalidade da base de dados;

- proposta de uma nova base de dados para classificação de e-mail legítimos utilizando dados de um servidor real e o filtro SpamAssassin.

Após esta introdução, a próxima seção apresenta a literatura relacionada. A Seção 3 descreve os métodos TEDA e FBeM para agrupamento de fluxos de dados e classificação de padrões, e o método de Spearman para redução do número de atributos. A Seção 4 investiga o desempenho dos classificadores evolutivos baseados em grânulos e nuvens de dados quando submetidos a uma grande base de dados real, coletada ao longo de 2 anos e 10 meses, contendo e-mails legítimos e spams. A conclusão e sugestões para trabalhos futuros se encontram na Seção 5 .

\section{TRABALHOS RELACIONADOS}

São consideradas técnicas anti-spam: (i) listas de bloqueio (bloqueio de mensagens de IPs contidos em listas negras); (ii) palavras-chave (bloqueio de mensagens contendo em seu corpo determinadas sequências de caracteres típicas em e-mails spam); (iii) recusa intencional (recusa em primeira instância, e adição do e-mail a uma Gray List, para posterior aceite em caso de re-envio); (iv) políticas de remetente (prevenção da substituição do cabeçalho do e-mail remetente); (v) técnicas baseadas em assinatura (o destinatário deve reconhecer a assinatura digital do remetente); e (vi) reconhecimento inteligente de padrões de spam. A última consiste essencialmente em modelos classificadores cujos parâmetros e estrutura são identificados a partir de um conjunto de amostras numéricas extraídas de e-mails e da combinação de algumas ou várias das técnicas mencionadas acima. Os modelos devem prover superfícies de separação entre as classes 'e-mail legítimo' e 'e-mail spam' em um espaço multidimensional.

O modelo classificador Naive Bayes (NB) é um dos mais usados em e-mails de texto (Schneider, 2003). Por ser um classificador simples e que apresenta boas taxas de detecções corretas de spam, NB é muito utilizado em sistemas comerciais de filtragem (Chen et al., 2008). Em (Frank et al., 2002), comparou-se três métodos baseados em NB, viz. Locally Weighted Naive Bayes (LWNB), Discretized Locally Weighted Naive Bayes (LWNBD) e Lazy Bayesian Rules (LBR), além de outros métodos como Averaged One-Dependence Estimator (AODE) e Knearest Neighbours With Distance Weighting (KNNDW). Propuseram-se melhorias e customizações no método NB original para tratar o problema de spam. Várias bases de dados de e-mails foram avaliadas, apontando os métodos $\mathrm{NB}$ modificados como aqueles com as maiores taxas de classificações corretas de spam.

Em (Braga \& Ladeira, 2007) foi apresentada uma abordagem que leva em consideração a característica dinâmica de spams, i.e., consideraram-se várias técnicas utilizadas por spammers para burlar sistemas anti-spam. Em préprocessamento, mensagens são transformadas e ordenadas em bases de dados numéricas utilizando o método Huffman Adaptativo baseado em árvore. Um dos benefícios da utilização de árvores adaptativas é a possibilidade de acrescentar novas folhas sem a necessidade de se criar uma nova árvore. Na classificação foi utilizado o método Support Vector Machine (SVM) (Cortes \& Vapnik, 1995), e a adaptação contou com uma técnica de envelhecimento exponencial. Em (Braga \& Ladeira, 2007), somente as mensagens mais recentes são consideradas. A abordagem considera que spams mudam ao longo do tempo. A ênfase é dada, então, às mensagens mais recentes.

Em (Soranamageswari \& Meena, 2011) enfatiza-se que modelos baseados em algoritmos de aprendizado offline 
usualmente falham quando uma nova mensagem spam é recebida, sendo que o sistema anti-spam já está em operação. É necessário reprojetar o modelo para que ele seja capaz de detectar o novo padrão. Porém, até que isso ocorra, o e-mail spam pode alcançar vários usuários. De uma maneira geral, os trabalhos procuram comparar modelos classificadores para verificar quais são os métodos de aprendizado e as estruturas de modelo mais eficientes (Guzella \& Caminhas, 2009). Contudo, tais métodos e modelos muitas vezes estão ao alcance dos spammers, que podem explorar suas vulnerabilidades.

Em (Guzella \& Caminhas, 2009) destaca-se que a aplicação de redes neurais artificiais (RNA) para detecção e filtragem de e-mails spam tem obtido melhores resultados que máquinas de vetor suporte, SVM. Adicionalmente, o método Random Forest (RF), que é constituído de um comitê de árvores de decisão, é discutido para a classificação supervisionada e semi-supervisionada de e-mails spam. Modelos RF têm apresentado melhor acurácia do que modelos NB, SVM e RNA (Guzella \& Caminhas, 2009).

Atualmente, estudos relacionados à classificação online de spam em e-mails de texto utilizando modelos adaptativos ao longo do tempo são raros. Os sistemas inteligentes evolutivos TEDA e FBeM, tratados na próxima seção, são abordagens apropriadas para ambiente não-estacionário, isto é, sujeitos à mudanças. Em outras palavras, modelos TEDA e FBeM são equipados com algoritmos incrementais para adaptação de seus parâmetros e estrutura de regras. Conforme novos padrões de spam surgem em um fluxo de dados online, a essência da informação é armazenada em novos modelos locais (nuvens ou grânulos). Ao mesmo tempo, os padrões identificados anteriormente são mantidos na memória. Os métodos e modelos mencionados nesta seção não possuem capacidade de evolução recursiva de parâmetros e estrutura de regras ao longo do tempo, conforme as propostas de TEDA e FBeM.

\section{CLASSIFICADORES EVOLUTIVOS}

Modelos classificadores evolutivos têm como principal característica a capacidade de adaptação a novidades (Ordóñez et al., 2013) (Bouchachia et al., 2007) (Iglesias et

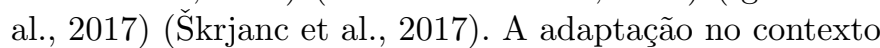
de fluxos de dados online é obtida a partir de algoritmos de aprendizado de máquina recursivos que visam melhorar gradativamente ou manter a acurácia do modelo em ambiente variante no tempo, i.e., em ambiente em que a distribuição que gera e governa os dados muda. Além disso, o processamento de dados de teste por parte de classificadores evolutivos pode se iniciar a partir de uma base de conhecimento vazia. Em outras palavras, os parâmetros de modelos locais e as propriedades dos dados não precisam ser conhecidos a priori. As abordagens TEDA e FBeM, tratadas no presente trabalho, são orientadas e foram customizadas para problemas de classificação em ambiente não-estacionário. Modelos de detecção de spam são obtidos sem a necessidade da introdução de conhecimento humano prévio sobre o domínio do problema de spam.

\subsection{TEDA Class}

TEDA é um método de modelagem baseado em regras capaz de lidar incrementalmente com grandes volumes de dados (Soares et al., 2018) (Angelov \& Yager, 2012). O aprendizado é baseado nos conceitos de excentricidade e tipicidade, relacionados às características de proximidade e densidade dos dados. O método se baseia em estimativas recursivas de densidade (Bezerra et al., 2016).

Seja $X=\left[x_{(1)}, \ldots, x_{(K)}\right]^{\prime}, X \in \Re^{K \times n}$, um conjunto de dados; e $k=1, \ldots, K$, o índice de tempo. A soma das distâncias, $\pi($.$) , da k$-ésima amostra, $x_{(k)}$, para todas as amostras de $X$ é dada por

$$
\pi\left(x_{(k)}\right)=\sum_{j=1}^{K} d\left(x_{(k)}, x_{(j)}\right)
$$

onde $d($.$) pode ser qualquer métrica de distância. Recur-$ sivamente, $\pi($.$) no índice de tempo k$, i.e., a soma das distâncias entre as $k$-ésimas amostras de dados e a amostra anterior de um fluxo de dados, é dada por

$$
\pi\left(x_{(k)}\right)=\pi\left(x_{(k-1)}\right)+d\left(x_{(k)}, x_{(k-1)}\right) .
$$

A excentricidade $\varepsilon($.$) de x_{(k)}$ em relação a todas as amostras contidas na base de dados é obtida de

$$
\varepsilon\left(x_{(k)}\right)=\frac{2 \pi\left(x_{(k)}\right)}{\sum_{j=1}^{K} \pi\left(x_{(j)}\right)} .
$$

A tipicalidade $\gamma($.$) é o complemento da excentricidade, i.e.,$

$$
\gamma\left(x_{(k)}\right)=1-\varepsilon\left(x_{(k)}\right)
$$

Uma regra TEDA é descrita por

$$
R_{i}(x): \text { Se }\left(x \sim x_{i}^{*}\right), \text { então Classe } z_{k}
$$

onde $i=1, \ldots, N$; e $\sim$ indica o relacionamento de uma amostra $x$ à regra $R_{i}$; e $x_{i}^{*}$ é um ponto representativo na nuvem de dados (ponto focal). Em particular, a regra $R_{i}$ mapeia $x \in \Re^{n}$ na classe $z_{k}$, que rotula a $i$-ésima nuvem de dados.

A excentricidade local, $\varepsilon_{i}$, e a tipicalidade local, $\gamma_{i}$, são definidas conforme as amostras são assimiladas à $i$-ésima nuvem de dados. Uma nuvem e uma regra correspondente são criadas quando o valor da tipicalidade local $\gamma_{i} \forall i$ é menor que um limiar $\alpha$, i.e.,

$$
\max _{i=1, \ldots, N} \gamma_{i}\left(x_{(k)}\right) \leq \alpha
$$

Caso contrário, a amostra $x_{(k)}$ é atribuída a regra mais ativa. O nível de ativação da $i$-ésima regra $R_{i}$ para uma $\operatorname{amostra} x_{(k)}$ é

$$
w_{i}\left(x_{(k)}\right)=\frac{\gamma_{i}\left(x_{(k)}\right)}{\sum_{j=1}^{N} \gamma_{j}\left(x_{(k)}\right)} .
$$

Assim que uma amostra é atribuida a uma regra $R_{i^{*}}$, a excentricidade local, $\varepsilon_{i^{*}}$, e a tipicalidade local, $\gamma_{i^{*}}$, são atualizadas. 
Detalhes da formulação TEDA, como um algoritmo geral, podem ser encontradas em (Soares et al., 2018) (Angelov \& Yager, 2012) (Bezerra et al., 2016). O algoritmo é sumarizado como segue.

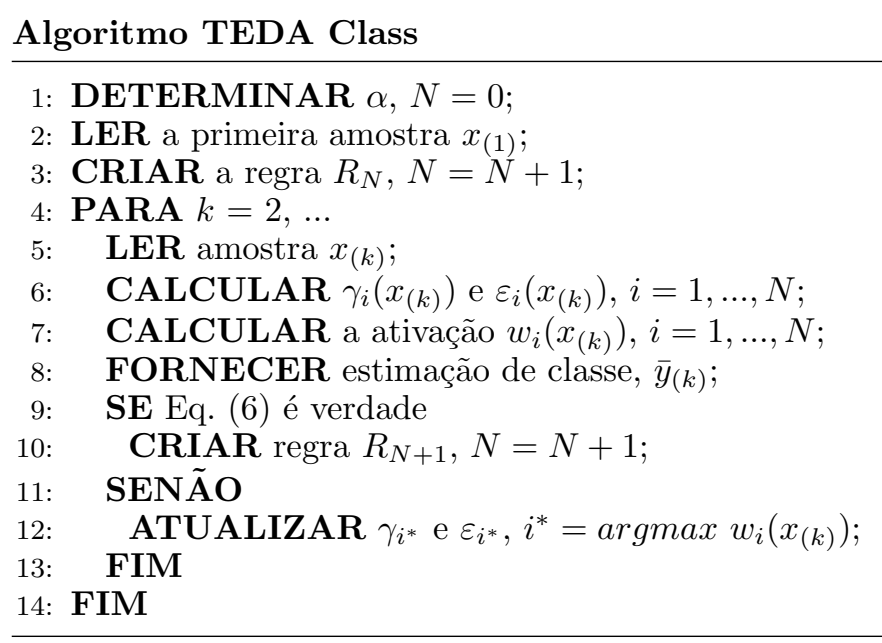

\subsection{FBeM Class}

O modelo FBeM consiste de um conjunto de regras fuzzy. FBeM é capaz de lidar com problemas online em que as amostras são ilimitadas e algoritmos offline convencionais apresentam problema de escalabilidade. FBeM é baseado em aprendizado incremental e fornece fronteiras não-lineares de separação de classes (Leite et al., 2013). O método não requer dados a priori para aprender. Regras e grânulos de informação fuzzy são criados dinamicamente e adaptados ao longo do tempo (Garcia et al., 2019). Para cada grânulo existe uma regra correspondente. A parte antecedente das regras FBeM consiste de hiper-retângulos fuzzy, e a parte consequente é uma classe basicamente. Neste trabalho, as regras FBeM têm a forma

$$
\begin{gathered}
R_{i}(x): \text { Se }\left(x_{1} \text { é } A_{1}^{i}\right) \mathrm{E} \ldots \mathrm{E}\left(x_{n} \text { é } A_{n}^{i}\right) \\
\text { Então }\left(\bar{y} \text { é Classe } z_{k}\right)
\end{gathered}
$$

sendo $x_{j}, j=1, \ldots, n$, a variável de entrada; $\bar{y}$ a classe estimada; e $R^{i}, i=1, \ldots, c$, a coleção de regras. $(x, y)^{[h]}$, $h=1, \ldots$ é um fluxo de dados; $y$ é a classe verdadeira. A classe verdadeira é conhecida somente após a estimação. Ademais, $A_{j}^{i}=\left(l_{j}^{i}, \lambda_{j}^{i}, \Lambda_{j}^{i}, L_{j}^{i}\right)$, são funções de pertinência trapezoidais criadas e desenvolvidas conforme os dados. A classe relativa à regra mais ativa para uma amostra $x^{[h]}$ é a saída global do modelo. A regra mais ativa é dada por

$$
\alpha^{*}=S\left(\alpha^{1}, \ldots, \alpha^{i}, \ldots, \alpha^{c}\right),
$$

onde considera-se o operador máximo, $\max _{i} \forall i$, como conorma triangular $S$. Além disso,

$$
\alpha^{i}=T\left(A_{1}^{i}, \ldots, A_{j}^{i}, \ldots, A_{n}^{i}\right),
$$

onde adotou-se o operador de agregação mínimo, $\min _{i} \forall i$, como norma triangular $T$.

A largura máxima que funções $A_{j}^{i} \forall i$ podem assumir ao longo do $j$-ésimo universo de discurso é limitada pela granularidade $\rho_{j}$. A largura de $A_{j}^{i}$ é dada por

$$
w d t\left(A_{j}^{i}\right)=L_{j}^{i}-l_{j}^{i}
$$

Logo,

$$
w d t\left(A_{j}^{i}\right) \leq \rho_{j}, i=1, \ldots, c .
$$

Diferentes valores de $\rho$ produzem diferentes representações do fluxo de dados em diferentes níveis de granularidade. A região de expansão de $A_{j}^{i}$ é denotada por

$$
E_{j}^{i}=\left[m p\left(A_{j}^{i}\right)-\frac{\rho_{j}}{2}, m p\left(A_{j}^{i}\right)+\frac{\rho_{j}}{2}\right]
$$

onde

$$
m p\left(A_{j}^{i}\right)=\frac{\lambda_{j}^{i}+\Lambda_{j}^{i}}{2}
$$

é o ponto central de $A_{j}^{i}$. A região $E_{j}^{i}$ auxilia na determinação de quando uma amostra $x^{[h]}$ deve ser considerada ou não como pertencente a um grânulo já existente. Para dados normalizados, o valor de $\rho$ varia em $[0,1]$. Se $\rho=1$, então o grânulo não é capaz de se expandir. Portanto, o processo de aprendizado cria uma regra para cada amostra de dados, o que pode levar ao overfitting e a um alto número de regras que não generalizam o comportamento dos dados. Se $\rho=0$, então cria-se um único grânulo para representar todos os dados, o que não é interessante em ambiente não não-estacionário. FBeM utiliza um procedimento rápido para evoluir $\rho$ ao longo do tempo.

Seja $r$ a diferença entre o número de grânulos atual e o número de grânulos a $h_{r}$ iterações passadas, i.e., $r=c^{[h]}-$ $c^{\left[h-h_{r}\right]}$. Se a quantidade de grânulos aumenta mais rápido do que uma taxa de crescimento $\eta$, ou seja, $r>\eta$, então $\rho$ é aumentado conforme

$$
\rho^{[h]}=\left(1+\frac{r}{h_{r}}\right) \rho^{\left[h-h_{r}\right]} .
$$

Isso controla o valor de $\rho$ e evita o aumento da complexidade do modelo. Se a granularidade $\rho$ cresce a uma taxa menor do que $\eta$, ou seja, $r<\eta$, então $\rho$ é reduzido,

$$
\rho^{[h]}=\left(1-\frac{\eta-r}{h_{r}}\right) \rho^{\left[h-h_{r}\right]} .
$$

A granularidade de modelos FBeM varia no tempo de acordo com o fluxo de dados. A criação de regras ocorre quando uma entrada $x=\left(x_{1}, \ldots, x_{n}\right)$ não pertence à região de expansão $\left(E_{1}^{i}, \ldots, E_{n}^{i}\right), i=1, \ldots, c$. Nesse caso,

$$
A_{j}^{c+1}=\left(l_{j}^{c+1}, \lambda_{j}^{c+1}, \Lambda_{j}^{c+1}, L_{j}^{c+1}\right)=\left(x_{j}, x_{j}, x_{j}, x_{j}\right),
$$

$j=1, \ldots, n$. O número de regras $c$ é acrescido de uma unidade.

A adaptação de parâmetros ocorre quando $x \in E^{i}$ para algum $i$. Existem seis possibilidades:

Se $x^{[h]} \in\left[m p\left(A_{j}^{i}\right)-\frac{\rho}{2}, l_{j}^{i}\right]$

Então $l_{j}^{i}(n e w)=x^{[h]}$ (expansão do suporte) 
Se $\left.\left.x^{[h]} \in\right] l_{j}^{i}, \lambda_{j}^{i}\right]$

Então $\lambda_{j}^{i}($ new $)=x^{[h]}$ (expansão do núcleo)

Se $\left.\left.x^{[h]} \in\right] \lambda_{j}^{i}, m p\left(A_{j}^{i}\right)\right]$

Então $\lambda_{j}^{i}(n e w)=x^{[h]}$ (contração do núcleo)

Se $\left.\left.x^{[h]} \in\right] m p\left(A_{j}^{i}\right), \Lambda_{j}^{i}\right]$

Então $\Lambda_{j}^{i}(n e w)=x^{[h]}$ (contração do núcleo)

Se $\left.\left.x^{[h]} \in\right] \Lambda_{j}^{i}, L_{j}^{i}\right]$

Então $\Lambda_{j}^{i}(n e w)=x^{[h]}$ (expansão do núcleo)

Se $\left.\left.x^{[h]} \in\right] L_{j}^{i}, m p\left(A_{j}^{i}\right)+\frac{\rho}{2}\right]$

Então $L_{j}^{i}(n e w)=x^{[h]}$ (expansão do suporte)

Procedimentos para remoção e mescla de grânulos e regras FBeM podem ser encontrados em (Leite et al., 2012). Procedimentos alternativos de mescla são reportados em (Škrjanc et al., 2019) (Soares et al., 2018). O aprendizado FBeM é sumarizado a seguir.

\section{Algoritmo FBeM Class}

: DETERMINAR $\rho, h_{r}, \eta$;

: PARA $h=1, \ldots$

RECEBER amostra $x^{[h]}$;

PROVER classe estimada $C^{[h]}$;

// A classe real se torna disponível

CALCULAR erro de estimação;

// Adaptação do modelo

SE $x^{[h]} \notin E^{i} \forall i$

CRIAR novo grânulo $\gamma^{[c+1]}$;

SENÃO

ADAPTAR grânulo mais ativo $\gamma^{i *}$;

\section{FIM}

REMOVER amostra $x^{[h]}$;

SE $h=\beta h_{r}, \beta=1, \ldots$

MESCLAR grânulos semelhantes;

ATUALIZAR a granularidade $\rho$;

REMOVER grânulos inativos;

\section{FIM}

FIM

\subsection{Correlação de Spearman para Seleção de Atributos}

A ordenação e seleção de atributos possibilitam a redução da complexidade de um problema. Um subconjunto de atributos mais relevantes ou discriminativos facilita a interpretação de um modelo, reduz a chance de overfitting, e pode produzir melhores resultados ao eliminarem variáveis que podem confundir o processo descoberta de padrões, tendências e relacionamentos. Seja $\left(x_{1}, \ldots, x_{i}, \ldots, x_{n}\right)$ um conjunto de atributos, e $y$ a variável dependente. Os atributos podem ser pontuados por

$$
S_{i}=\frac{1}{n} \sum_{j=1}^{n}\left|\rho\left(x_{i}, x_{j}\right)-\rho\left(x_{i}, y\right)\right|,
$$

sendo $i=1, \ldots, n ; \rho$ é o coeficiente de Spearman nesta sub-seção (Soares et al., 2018),

$$
\rho\left(x_{i}, x_{j}\right)=1-\frac{6 \sum_{k=1}^{K} d_{i j(k)}^{2}}{K\left(K^{2}-1\right)},
$$

sendo $K$ o número de amostras. Além disso,

$$
d_{i j(k)}^{2}=\operatorname{rank}\left(x_{i(k)}\right)-\operatorname{rank}\left(x_{j(k)}\right)
$$

é a diferença entre as classificações das amostras após a ordenação destas de forma ascendente. Se valores consecutivos de uma variável, $x_{i(k)}$ e $x_{i(k+1)}$, são idênticos, então valores fracionários, iguais à média de suas posições na ordem ascendente de valores, são atribuídos. Isto é equivalente a uma média sobre todas as permutações possíveis.

O $\rho$ de Spearman varia de -1 a 1 . Um valor de 1 (ou -1) implica que uma função monotonamente crescente (decrescente) descreve a relação entre as variáveis perfeitamente. Uma correlação de 0 indica que não há tendência do valor de uma variável aumentar ou diminuir quando outra variável aumenta. O coeficiente de Spearman aumenta conforme as variáveis tornam-se mais próximas de serem funções monótonas perfeitas umas das outras. Diferentemente do coeficiente de Pearson (Bishop, 1995), o $\rho$ de Spearman não assume linearidade ou normalidade.

Quanto mais próximo de 0 é o valor de $S_{i} \in[-1,1]$, mais importante é o $i$-ésimo atributo. A idéia é que um atributo interessante possui baixa correlação com outros atributos, o que implica que este transmite informações únicas e diferentes. Uma alta correlação com a variável dependente indica que um atributo auxilia na previsão. Atributos são deixados de fora do modelo conforme suas pontuações $S_{i}$. Quando um atributo gera uma redução significativa de desempenho do modelo, então o processo de eliminação de novos atributos da lista $S_{i}$ é encerrado.

Como a velocidade de processamento é essencial em ambiente online, o método de seleção de atributos descrito nesta seção é útil para reduzir o problema de dimensionalidade.

\section{METODOLOGIA}

A base de dados considerada neste trabalho foi obtida a partir de 25745 e-mails enviados para o endereço $<$ cemes@cemes.edu.br> entre julho de 2014 e abril de 2017. O filtro anti-spam SpamAssassin, disponível em $<$ http://spamassassin.apache.org/>, foi utilizado para verificar a veracidade dos e-mails. O SpamAssassin é um filtro de spam de código aberto amplamente utilizado. Ele é composto de 711 testes que verificam a presença de palavras-chave contidas no corpo do e-mail e realizam testes de cabeçalho e URL (Uniform Resource Locator). Por exemplo, o teste 584 verifica se o nome do remetente contém nomes de drogas. Já o teste 362 verifica se o endereço do remetente consta em uma 'lista negra'.

Cada teste realizado é associado à uma pontuação. Quando padrões de spam são identificados, um valor numérico maior do que 0 é atribuído ao teste. Quanto maior é o valor de um teste, maior é a chance de que o e-mail em questão seja spam. A pontuação dos 711 testes é somada. Caso a soma seja maior ou igual a um limiar, cujo valor padrão é 5 , então o e-mail é definido como spam. Caso contrário, o e-mail é considerado legítimo. 
A partir da API disponível em < http://spam check.postmarkapp.com/> carregou-se os 25745 e-mails coletados e obteve-se uma pontuação nos 711 testes SpamAssassin para cada um. O resultado indicou a existência de 7830 e-mails spam e 17915 e-mails legítimos. Os dados obtidos nos 711 testes individuais foram normalizados na escala $[0,1]$ para que as variáveis fossem consideradas na mesma proporção. A normalização do $i$-ésimo elemento do $k$-ésimo vetor de dados é dada por

$$
x_{i(k)}^{r}=\frac{x_{i(k)}-\min _{\forall j}\left(x_{i(j)}\right)}{\max _{\forall j}\left(x_{i(j)}\right)-\min _{\forall j}\left(x_{i(j)}\right)},
$$

sendo $j=1, \ldots, k-1$; e $x_{i(k)}^{r} \in[0,1]$.

Classificação de fluxo de dados envolve pares $(x, C)^{[h]}$, $h=1, \ldots$ Assume-se $C$ desconhecido em princípio e, portanto, algoritmos de aprendizado não-supervisionados. Não-estacionariedades ao longo do tempo podem ser capturadas devido a adaptação incremental dos classificadores evolutivos TEDA e FBeM. Logo, a fronteira discriminativa de classes de ambos é variável no tempo.

A acurácia dos classificadores é calculada conforme

$$
A c c(\%)=\frac{V P+V N}{V P+F P+V N+F N},
$$

onde $V P, F P, V N$, e $F N$ referem-se a verdadeiros e falsos, positivos e negativos. A classe negativa (classe 0 ) indica e-mail legítimo. A classe positiva (classe 1) referese à e-mails spam. Ademais, utilizou-se também a área sob a curva ROC (Receiver Operating Characteristic) para análise da acurácia de classificação (Duda et al., 1995). A análise ROC fornece um método conveniente para avaliar a qualidade dos classificadores evolutivos, pois é insensível às mudanças nas distribuições das classes e à proporção de amostras por classe. A área ROC é definida a partir das taxas de $V P$ e $F P$, conforme

$$
\begin{aligned}
V P_{\text {taxa }} & =\frac{V P}{V P+F N}, \\
F P_{\text {taxa }} & =\frac{F P}{F P+V N} .
\end{aligned}
$$

Logo,

$$
A=\int_{0}^{1} V P_{t a x a}(t) F P_{t a x a}(t) d t,
$$

onde $A$ é a área sob a curva ROC; $t$ é o tempo; $V P_{t a x a}$ e $F P_{\text {taxa }}$ são as taxas de verdadeiro-positivo e falso-positivo, respectivamente.

Para cada classe, a análise ROC aplica um valor limiar no intervalo [0,1] para a saída. Quanto mais a curva ROC se aproxima do limite superior esquerdo do gráfico $V P_{\text {taxa }} \times$ $F P_{\text {taxa }}$, melhor é o desempenho do classificador.

Os parâmetros iniciais do classificador FBeM são $\rho=0.06$; $h_{r}=10000 ; \eta=1$; e TEDA usa $m=2$. Estes valores foram obtidos por tentativa e têm se mostrado apropriados em diferentes simulações. O classificador Naive Bayes também foi avaliado. NB utilizou os parâmetros estabelecidos como padrão. O método para seleção de atributos baseado na correlação de Spearman foi empregado. Os modelos classificadores foram avaliados a partir dos 711 atributos originais, e também a partir de $75 \%, 50 \%$ e $25 \%$ dos atributos dentre aqueles indicados como os mais discriminativos de classes. O número de regras (modelos locais - grânulos ou nuvens) desenvolvido pelos diferentes classificadores, assim como o tempo de processamento, também são considerados nas comparações. Os experimentos foram realizados no ambiente Matlab 2018a, em computador com sistema operacional macOS High Sierra, Intel Core i5 com 1,8 GHz e 8GB de memória RAM DDR3.

\section{RESULTADOS}

Simulações computacionais foram realizadas para avaliar a acurácia dos modelos evolutivos FBeM e TEDA, e também do modelo mais comum na área, Naive Bayes, combinados com o método de seleção de atributos utilizando o seletor de atributos baseado na correlação de Spearman. A Tabela 1 sumariza os resultados obtidos para diferentes conjuntos de atributos. O número de regras ou modelos locais desenvolvidos por FBeM e TEDA foram controlados por meio dos parâmetros $\rho$ e $m$, respectivamente, para que não mais de 10 modelos locais fossem desenvolvidos. Esta abordagem é útil para uma comparação justa. Maior acurácia pode ser obtida conforme o número de grânulos e nuvens aumenta, ou seja, a partir do uso de classificadores com maior estrutura e parâmetros. O modelo NB não retorna nenhum tipo de regra.

Nota-se na Tabela 1 que os classificadores são mais precisos quando os 711 atributos são considerados. Informações são perdidas quando certos atributos não são utilizados. Entretanto, excluem-se os atributos menos relevantes a partir do método de correlação de Spearman. O classificador granular FBeM obteve maior acurácia do que o classificador TEDA baseado em nuvens em todos os casos. Um número relativamente grande de atributos pôde ser removido sem perda significativa de acurácia. Logo, o método baseado na correlação de Spearman tem um papel fundamental na redução da dimensão do espaço. Como conseqüência, um menor tempo total de processamento é percebido com a redução do número de atributos. O tempo de processamento é um fator importante em modelagem online. Por exemplo, um servidor de e-mail deve processar milhares de e-mails por segundo. Ressalta-se que neste contexto, algoritmos de agrupamento offline, como o Naive Bayes, K-Means, Fuzzy C-Means, Gustafson-Kessel e Gath-Geva, são infactíveis, pois precisam realizar múltiplas análises (épocas de treinamento) sobre um conjunto de e-mails.

O melhor resultado apresentado na Tabela 1 depende de um compromisso entre a capacidade de processamento disponível no servidor e a acurácia de classificação. Notase na Tabela 1, que a capacidade adaptativa de FBeM e TEDA permite que estes captem as mudanças provenientes da fonte geradora de dados. Isto faz com que estes modelos apresentem melhor desempenho em termos de acurácia quando comparados à modelos não-evolutivos, como o modelo NB. Nota-se também vantagem dos modelos evolutivos em tempo de processamento comparados ao modelo NB, pois estes não precisam ser re-treinados. Valores em 
negrito e sublinhados na Tabela 1 representam o melhor desempenho no critério em questão.

Tabela 1. Desempenho comparativo dos modelos classificadores FBeM, TEDA e Naive Bayes

\begin{tabular}{c|cccc}
\hline- & \multicolumn{5}{|c}{ FBeM } \\
\hline \# Atributos & Acc (\%) & Tempo (s) & \# Regras & A-ROC \\
\hline 711 & $\mathbf{9 4 , 7 8}$ & 137,2 & 10 & $\mathbf{0 , 9 5 1}$ \\
533 & 91,32 & 100,1 & 7 & 0,924 \\
355 & 90,91 & 89,7 & 7 & 0,912 \\
178 & 81,47 & 73,7 & $\underline{\mathbf{}}$ & 0,833 \\
\hline- & \multicolumn{5}{|c}{ TEDA } \\
\hline \# Atributos & Acc $(\%)$ & Tempo (s) & \# Regras & A-ROC \\
\hline 711 & 93,89 & 92,3 & 10 & 0,944 \\
533 & 89,95 & 89,6 & 10 & 0,905 \\
355 & 88,97 & 73,0 & 10 & 0,899 \\
178 & 83,47 & $\mathbf{5 7 , 3}$ & 10 & 0,854 \\
\hline- & \multicolumn{5}{|c}{ Naive Bayes } & \\
\hline \# Atributos & Acc $(\%)$ & Tempo (s) & \# Regras & A-ROC \\
\hline 711 & 86,57 & 188,1 & - & 0,872 \\
533 & 85,94 & 180,5 & - & 0,864 \\
355 & 85,42 & 124,4 & - & 0,859 \\
178 & 84,49 & 82,2 & - & 0,856 \\
\hline
\end{tabular}

As matrizes de confusão dos melhores casos de acurácia de FBeM e TEDA, i.e., usando os 711 atributos originais, são mostrados na Fig. 1. É importante observar alguns pontos. Primeiro, a classificação FBeM é superior a classificação TEDA em todos os quadrantes. Isto se deve essencialmente à particularidades dos algoritmos de aprendizado e da estrutura de suas regras. As áreas sob as curvas ROC (conforme Tabela 1) também demonstram uma maior eficiência de classificação do modelo FBeM com relação ao modelo TEDA. No melhor cenário, FBeM tem área ROC igual a 0,951, enquanto TEDA apresenta área 0,944.

Outro ponto é que ambas as matrizes de confusão indicam um melhor desempenho de classificação de FBeM e de TEDA para a classe 0, i.e., 'e-mails legítimos'. Isto se deve ao fato de que e-mails spam mudam de padrão ao longo do tempo. Note que as amostras foram coletadas em um período de 2 anos e 10 meses em um ambiente real. As fronteiras de separação de classes de ambos os modelos classificadores, FBeM e TEDA, são adaptadas após algumas iterações e erros, porém a variação temporal de padrões spam justifica o erro ligeiramente maior para e-mails spam. Um terceiro ponto a se notar é que os quadrantes da diagonal secundária da Figura 1 (em vermelho) são indicadores de erro. Em geral, considera-se ser um erro mais grave indicar que um e-mail é spam quando se trata de um e-mail legítimo já que os usuários não costumam verificar a caixa de spam. Do contrário, se um email spam não for filtrado, o usuário ainda pode reconhecer que se trata de um spam. Há um risco associado, porém a exclusão de um e-mail importante pode ser uma falha mais significativa. Nesse aspecto, em 2,3\% dos casos, FBeM indicou erroneamente que um e-mail legítimo era spam, enquanto TEDA fez o mesmo em 3,0\% dos casos.

Várias mensagens legítimas possuem atributos que se confundem com mensagens spam. Por exemplo, se o usuário assinou uma lista de e-mails em um site de comércio eletrônico, este, por sua vez, pode enviar mensagens contendo promoções, imagens, HTML, o que confunde mecanismos

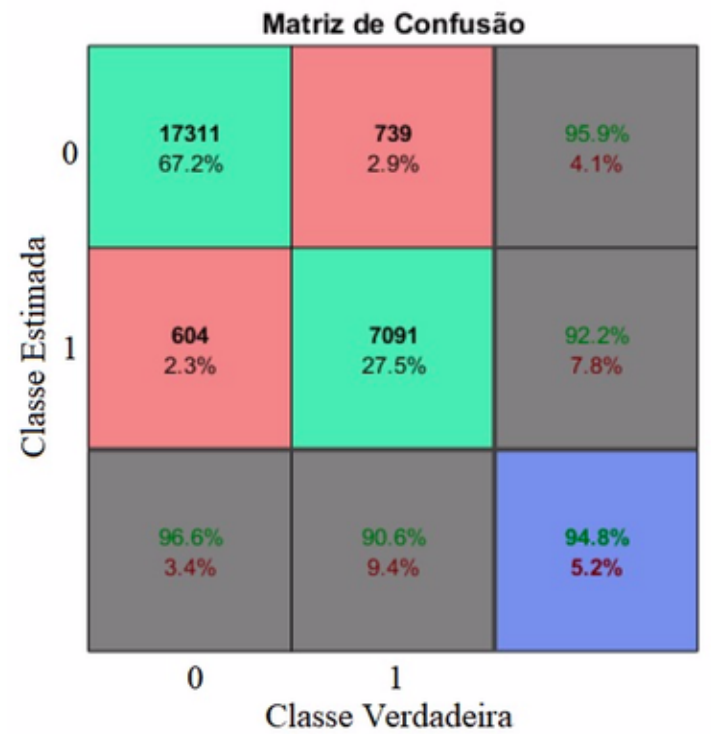

(a)

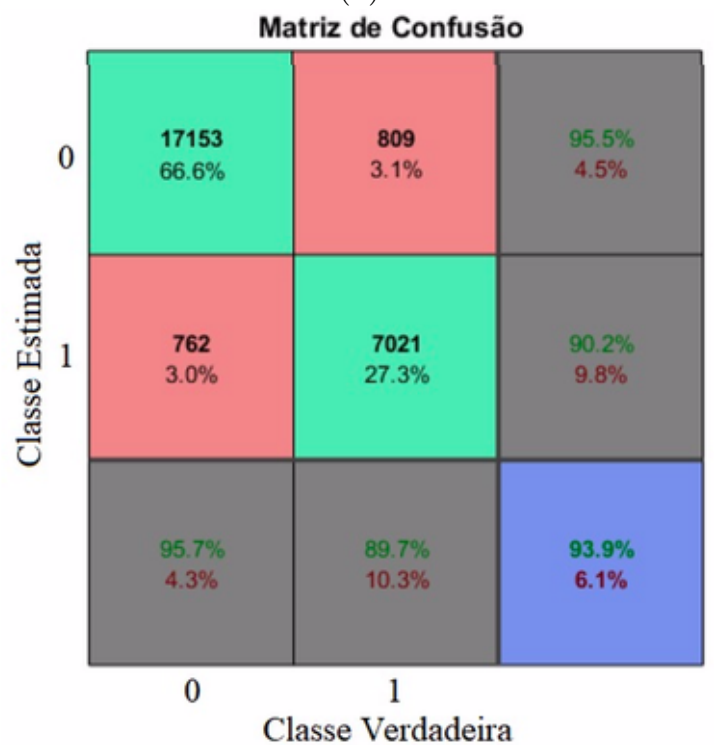

(b)

Figura 1. Matriz de Confusão: (a) FBeM, (b) TEDA

de classificação e até mesmo a nossa noção intuitiva do que é um e-mail spam, dado o conteúdo generalista de um e-mail que contém promoções e links para sites externos. Desta forma, conclui-se que a acurácia de ambas as abordagens, FBeM e TEDA, são 'muito boas' e, acima disso, ambas são factíveis para lidar com o problema de variação temporal de padrões de mensagens spam.

\section{CONCLUSÃO}

Classificadores inteligentes evolutivos baseados em grânulos fuzzy, FBeM, e nuvens de dados, TEDA, foram propostos para classificação não-supervisionada de e-mails spam e de e-mails legítimos. Ademais, um método nãoparamétrico de seleção de atributos baseado na correlação de Spearman foi empregado para redução da dimensionalidade das amostras. 
Experimentos mostraram que os métodos evolutivos foram eficientes na classificação não-supervisionada, apresentando acurácias entre 93\% e 95\% nos melhores casos, superando o modelo Naive Bayes, comumente utilizado na área. Isso é possível visto que os primeiros são capazes de acompanhar as mudanças da fonte geradora de dados. A análise de matrizes de confusão mostrou maior dificuldade na classificação de instâncias spam. Isto ocorre devido às mudanças na fonte. Em outras palavras, as técnicas utilizadas por spammers variam no tempo. O método FBeM foi ligeiramente superior ao método TEDA em acurácia, conforme comprovado em análise ROC. No entanto, o método TEDA demonstrou maior velocidade para processar os dados de e-mails, o que pode ser determinante em um ambiente de fluxo de dados em alta freqüência. Em 2,3\% dos casos, FBeM indicou erroneamente que um e-mail legítimo era spam, contra 3,0\% de indicações equivocadas de TEDA - sendo esta a situação mais crítica.

Os índices de erro, tanto de FBeM quanto de TEDA, são relativamente muito baixos nesta aplicação. Ambos os métodos evolutivos são adequados para serem utilizados na classificação de spam, visto que os atributos de e-mails spam são modificados ao longo do tempo para lubridiar usuários e sistemas. Essas mudanças nos atributos podem ser assimiladas por abordagens online. Abordagens offline requerem re-treinamento do modelo anti-spam, o que pode ser custoso e válido por curto prazo.

Trabalhos futuros considerarão seleção incremental de atributos e diferentes variáveis estatísticas associadas às regras FBeM e nuvens TEDA. Por exemplo, será avaliada a utilidade de capturar e manter informações sobre especificidade, entropia, correntropia e cardinalidade associadas às amostras de e-mails. Estas variáveis podem ser úteis na otimização estrutural dos modelos evolutivos durante o aprendizado online. Processamento de dados incertos e análise de spam em imagens serão tópicos considerados.

\section{AGRADECIMENTOS}

Este trabalho recebeu apoio do Instituto Serrapilheira, número do processo Serra - 1812-26777. O último autor agradece à Fundação de Amparo à Pesquisa do Estado de Minas Gerais (FAPEMIG), processo APQ-03384-18.

\section{REFERÊNCIAS}

Angelov, P., Yager, R. A new type of simplified fuzzy rule-based system. Int Journal of General Systems, vol. 41(2), pp. 163-185, 2012.

Bezerra, C., Costa, B., Guedes, L. A, Angelov, P. An evolving approach to unsupervised and real-time fault detection in industrial processes. Expert Syst Appl, vol. 63, pp. 134-144, 2016.

Bishop, C. Neural Networks for Pattern Recognition. Oxford University Press, Oxford, 1995.

Bouchachia, A., Gabrys, B., Sahel, Z. Overview of some incremental learning algorithms. In: IEEE Int Conf on Fuzzy System, pp. 6p., 2007.

Braga, I., Ladeira, M. Um modelo adaptativo para a filtragem de spam. In: XXVII Congresso da Sociedade Brasileira de Computação, pp. 1381-1390, 2007.

Chen, C., Tian, Y., Zhang, C. Spam filtering with several novel Bayesian classifiers. In: IEEE Int Conf on Pattern Recognition, pp. 4p., 2008.
Cortes, C., Vapnik, V. Support vector networks. Machine Learning, vol. 20(3), pp. 273-297, 1995.

Duda, R. O., Hart, P. E., Stork D. G. Pattern Classification. John Wiley \& Sons, 2012.

Fossi, M., Egan, G., Haley, K., Johnson, E., Mack, T., Adams, T., Blackbird, J., Low, M. K., Mazurek, D., McKinney, D. Symantec internet security threat report trends for 2010. Symantec Report, vol. XVI, 2011.

Frank, E., Hall, M., Pfahringer, B. Locally weighted naive bayes. In: 19th Conf on Uncertainty in Artificial Intelligence, Morgan Kaufmann Pub., pp. 249-256, 2002.

Garcia, C., Leite, D., Škrjanc, I. Incremental MissingData Imputation for Evolving Fuzzy Granular Prediction. IEEE Trans on Fuzzy Syst, pp. 1-15, 2019, DOI: 10.1109/TFUZZ.2019.2935688.

Guzella, T., Caminhas, W. A review of machine learning approaches to spam filtering. Expert Syst Appl, vol. 36(7), pp. 10206-10222, 2009.

Iglesias, J. A., Ledezma A., Sanchis A, Angelov, P. RealTime recognition of calling pattern and behaviour of mobile phone users through anomaly detection and dynamically-evolving clustering. Applied Sciences, vol. $7(8)$, pp. 1-14, 2017.

Leite, D., Ballini, R., Costa, P., Gomide, F. Evolving fuzzy granular modeling from nonstationary fuzzy data streams. Evolving Systems, vol. 3(2), pp. 65-79, 2012.

Leite, D., Costa, P., Gomide, F. Evolving granular neural networks from fuzzy data streams. Neural Networks, vol. 38, pp. 1-16, 2013.

Olivo, C. K., Santin, A. O., Oliveira, L. E. Abordagens para detecção de spam de e-mail. In: XV Simpósio Brasileiro em Segurança da Informação e de Sistemas Computacionais, vol. 1, pp. 141-182, 2015.

Ordóñez, F., Iglesias, J., Toledo, P., Ledezma, A., Sanchis, A. Online activity recognition using evolving classifiers. Expert Syst Appl, vol. 40(4), pp. 1248-1255, 2013.

Schneider, K.-M. A comparison of event models for naive bayes anti-spam e-mail filtering. In: 10th European Conference of the Association for Computational Linguistics, pp. 307-314, 2003.

Škrjanc, I., de Miguel, A. S., Iglesias, J. A., Ledezma, A., Dovžan, D. Evolving Cauchy possibilistic clustering based on cosine similarity for monitoring cyber systems. In: IEEE Conf Evolving Intell Syst, pp. 6p., 2017.

Škrjanc, I., Iglesias, J., Sanchis, A., Leite, D., Lughofer, E., Gomide, F. Evolving fuzzy and neuro-fuzzy approaches in clustering, regression, identification, and classification: A survey. Info Sci, vol. 490, pp. 344-368, 2019.

Soares, E., Costa, P., Costa, B., Leite, D. Ensemble of evolving data clouds and fuzzy models for weather time series prediction. Appl Soft Comput, vol. 64, pp. 445-453, 2018.

Soares, E., Camargo, H., Camargo, S., Leite, D., Incremental gaussian granular fuzzy modeling applied to hurricane track forecasting. In: IEEE Int Conf on Fuzzy System, pp. 8p., 2018.

Soranamageswari, M., Meena, C. A novel approach towards image spam classification. Int Journal of Comput Theory and Eng, vol. 3(1), pp. 84-88, 2011.

Whitworth, B., Whitworth, E. Spam and the socialtechnical gap. IEEE Comput, v. 37(10), pp. 38-45, 2004. 